\title{
Forgetting Curve on Basic Cardiopulmonary Resuscitation Competence in Simulation Models
}

\author{
Moises Natanael DISR*, Eduardo GL, Ramón IEH, Gillberto SB, Pablo \\ MGM and Laura REA \\ Facultad de Medicina, Universidad Autonoma de Yucatan, Mexico
}

*Corresponding author: Moisés Natanael de los Santos Rodríguez, Facultad de

Medicina, Universidad Autonoma de Yucatan, Mexico, Tel: 01 9999233260; E-mail: moysantos86@gmail.com

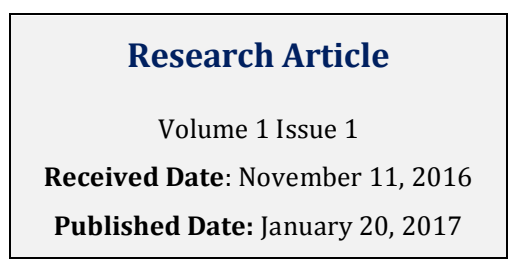

\section{Abstract}

Introduction: Simulation model based medical education considerably improves skills on health professionals to perform high-quality cardiopulmonary resuscitation (CPR); nevertheless recent studies report low competence for CPR in latter-year students, which might be explained due to loss of skill due to the time lapse between training and actual experience.

Method: This was a cohort study, performed at UADY School of Medicine's Simulation Laboratory. 27 third-year (preclinical, group A) and 31 fifth-year (clinical, group B) students were included. Both groups received the same training by the same instructor during two weeks, and were periodically evaluated over a 3-month period.

Results: Forgetting occurred in 40 (69\%) students; 2 (7.4\%) of group A by the first month, 14 (51.9\%) by the second month, and $19(70.4 \%)$ by the third month. On group B, forgetting occurred in 3 (9.7\%) students by the first month, 18 (58.1\%) by the second month, and $21(67.7 \%)$ by the third month. No significant differences were found between groups $(\mathrm{p}=0.992)$.

Conclusion: Competence for basic CPR in simulation models is lost within 3 months after training. There were no statistically significant differences between students already in clinical practice and those who have not started clinical practice.

Keywords: Forgetting curve; Basic cardiopulmonary resuscitation; Simulation models

\section{Introduction}

Simulation-based medical education (SBME), has progressively become an effective method to teach clinical skills in undergraduate students and health professionals [1,2]. SBME helps students to gain confidence to perform processes in early stages of their instruction, which favors a less stressful and more productive transition to hospital practice [3]. Cardiac arrest is currently one of the main causes of death worldwide, and it is potentially reversible if timely treated [4]. Immediate delivery of high-quality basic cardiopulmonary resuscitation (BCPR) is crucial for 


\section{Open Access Journal of Pharmaceutical Research}

outcome of patients on cardiac arrest. Survival rates fall circa $10 \%$ for every minute without BCPR, whilst risk of permanent brain injury substantially increases; on BCPR, fall of survival rates is limited to $4 \%$, justifying international guidelines recommending routine training on BCPR for health professionals $[5,6]$.

It has been shown that practice on simulation models substantially improves skills for high-quality BCPR on health professionals [7]. In spite of this, recent studies report low competence for BCPR on medical students, which might be a consequence of skill loss due to the time lapse between training and actual experience, which can occur as soon as 3 months after training [8-10]. A possible explanation of the difficulty to perform skills learned in simulation on real patients is the time lapse until students face the opportunity to use acquired skills in a clinical scenario; this occurs in students who have not started hospital-based clinical practice. As a result of this, permanent gain of skills and patient safety might be compromised [8]. Due to the fact that every training has a time-related forgetting, and considering that BCPR skills rapidly decline, it should not be assumed that these skills will remain indefinitely, but rather it is recommended to determine the time in which these skills should be retrained [4]. The curricula for Medicine at Universidad Autónoma de Yucatán (UADY) medical school includes, in its fifth year, a two-month training on airway management and advanced cardiopulmonary resuscitation. We proposed to identify the most frequent mistakes amongst the loss of competence for BCPR and the forgetting curve on UADY medical students for BCPR on simulation models, in order to guide curricular amendments that guarantee competence in BCPR during their practice in clinical scenarios.

\section{Methods}

We performed a longitudinal, prospective, analytic observational study on two cohorts of medical students, cycle 2013-2014. Sample size was calculated with Epi-info 6.0 considering an alpha of 0.5 , beta of 0.80 , and a universe of 140 students, estimating a forgetting of the skills acquired via simulation of $60 \%$ in the group without hospital practice and $30 \%$ in the group with hospital practice. The minimum sample size resulted on 48 subjects with a $1: 1$ rate. We included 27 third-grade students (group A) and 31 fifth-grade students (group B), all of which had no previous formal training in BCPR, who voluntarily signed an informed consent, and then underwent training in BCPR in simulation models. These two groups were included to control for exposition to clinical training in a hospital scenario and experiences in
CPR derived from this. Both groups received the same training by the same instructor, in order to avoid bias in learning experience. The training comprised three phases:

a. Phase 1, Training: This included a review of the literature on BCPR, according to the guidelines published on 2010 by the American Heart Association (AHA) $[8,10]$; then, the students had a practice session of BCPR in high-fidelity simulation models under supervision, and a debriefing afterwards.

b. Phase 2, Practice: Both groups had two weeks to practice as many times as they deemed necessary to acquire competence in BCPR. They had a debriefing after every practice.

c. Phase 3, Evaluation: After the 2 weeks of practice, each student participated in a simulated clinical scenario with a high-fidelity model, without debriefing. The performance was recorded and then evaluated with a checklist [11] by an AHA-certified Basic Life Support instructor. This procedure was repeated after one, two and three months.

d. In order to identify whether a statistically significant difference in competence on BCPR existed at different time points, we used McNemar test with a significance of $95 \%(\mathrm{p}<0.05)$.

e. For statistical analysis of the forgetting curve we performed a monthly survival analysis represented in a Kaplan-Meier curve, in which "death" represented forgetting and "survivors" were censored, meaning they did not show forgetting during the study. We compared both forgetting curves with the Log Rank test. Statistical analysis was done on SPSS Statistics ${ }^{\circledR}$ version 20.

f. This study was approved by the Graduate and Postgraduate studies committee of UADY medical school. Ethical considerations were done following the Mexican Technical Norm \#313 for presentation of research projects and technical reports in health institutions. There was no conflict of interest or external financing. None of the researchers profited from this research.

\section{Results}

Between August and September 2013, 65 students were invited to participate in the research, among which 58 met eligibility criteria. Of these, 35 (60.3\%) were male, and 23 (39.7\%) female. Participants were then divided in 2 groups: Group A (3rd year), with 27 (46.5\%), and Group B (5th year), with 31 (53.5\%) medical school students. In 


\section{Open Access Journal of Pharmaceutical Research}

order to determine competence for BCPR through time, we used a Kaplan-Meier survival curve, in which, in Group A, at month 0 , one student (3.7\%) did not achieve competence for BCPR. Loss of competence (forgetting) was observed in 2 students (7.4\%) at month 1,14 students (51.9\%) at month 2, and 19 students (70.4\%) at month 3. In Group B, 31 students (100\%) achieved competence at month 0 , among which loss of competence was observed in 3 students $(9.7 \%)$ at month 1,18 students $(58.1 \%)$ at month 2 , and 21 students $(67.7 \%)$ at month 3 (Table 1).

\begin{tabular}{|c|c|c|c|c|c|c|}
\hline \multirow{2}{*}{ Group } & \multirow{2}{*}{$\begin{array}{c}\text { Time } \\
\text { (months) }\end{array}$} & \multirow{2}{*}{ State } & \multicolumn{2}{|c|}{ Survival } & \multirow{2}{*}{$\begin{array}{c}\text { No } \\
\text { competence } \\
\text { for CPR }\end{array}$} & \multirow{2}{*}{$\begin{array}{l}\text { Competence } \\
\text { for CPR }\end{array}$} \\
\hline & & & Estimation & Tipical mistakes & & \\
\hline \multirow{4}{*}{ A } & 0 & forget & 0.963 & 0.036 & $1(3.7 \%)$ & $26(96.3 \%)$ \\
\hline & 1 & forget & 0.926 & 0.05 & $2(7.4 \%)$ & $25(92.6 \%)$ \\
\hline & 2 & forget & 0.481 & 0.096 & $14(51.9 \%)$ & $13(48.1 \%)$ \\
\hline & 3 & forget & 0.296 & 0.088 & $19(70.4 \%)$ & $8(29.6 \%)$ \\
\hline \multirow{4}{*}{ B } & 0 & censored & 0.1 & 0 & $0(0 \%)$ & $31(100 \%)$ \\
\hline & 1 & forget & 0.903 & 0.053 & $3(9.7 \%)$ & $28(90.3 \%)$ \\
\hline & 2 & forget & 0.419 & 0.089 & $18(58.1 \%)$ & $13(41.9 \%)$ \\
\hline & 3 & forget & 0.323 & 0.084 & $21(67.6 \%)$ & $10(32.4 \%)$ \\
\hline
\end{tabular}

Table 1: Survival table Kaplan-Meier.

\begin{tabular}{|c|c|c|c|c|c|}
\hline \multicolumn{2}{|c}{ c } & \multicolumn{2}{c|}{ After } & \multirow{2}{*}{ Total } \\
\cline { 3 - 6 } \multicolumn{2}{|c|}{} & CPR Competence & CPR No Competence & 57 \\
\hline \multirow{3}{*}{ Before } & CPR Competence & Frequency & 18 & 39 & $98.3 \%$ \\
\cline { 2 - 6 } & & $\%$ & $31.0 \%$ & $67.2 \%$ & 1 \\
\hline \multirow{3}{*}{ Total } & CPR & Frequency & 0 & 1 & $1.7 \%$ \\
\cline { 2 - 6 } & No Competence & $\%$ & $0.0 \%$ & $1.7 \%$ & 58 \\
\hline & & Frequency & 18 & $69.0 \%$ & $100.0 \%$ \\
\hline
\end{tabular}

Table 2: CPR Competence Evalution. Test de McNemar.

Forgetting occurred in 40 students (69\%); 19 in Group A, 21 in Group B by 3 months. In order to know whether forgetting observed in students among the 2 groups was significant we performed the McNemar test, which showed a p-value of 0.001 , thus rejecting null hypothesis, which established that competence for BCPR remains 3 months after training (Table 2). When we compared forgetting curves in the 2 groups with Log-Rank test, no significant difference was found at any time point $(\mathrm{p}=0.992)$ (Figure 1).

Figure1: Curve forget competition for basic CPR in both groups.

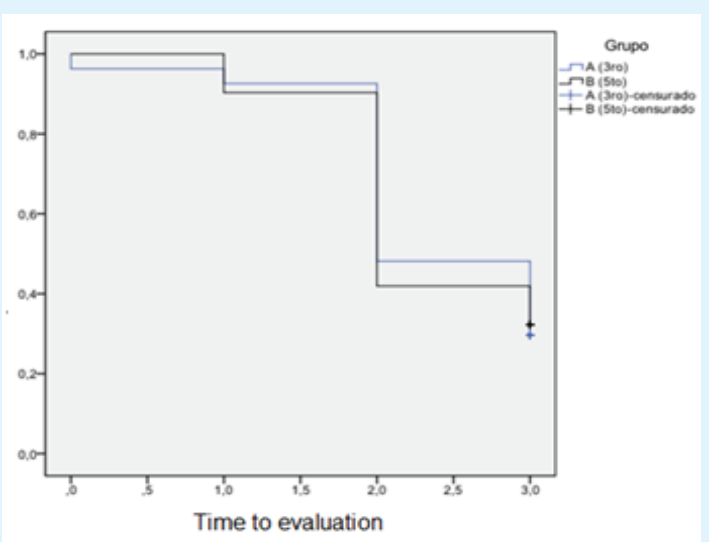




\section{Open Access Journal of Pharmaceutical Research}

Finally, in order to determine abilities lost through time, we registered mistakes observed in students throughout the study; among these, the most common mistake was related to ventilation, observed in $6(22.2 \%)$ Group A and 10 (32.3\%) Group B students, followed by fail to verify pulse, observed in 5 (18.5\%) Group A and 6 (19.4\%) Group B students. Third most common mistake was with diagnosis of cardiac arrest, in 5 (18.5\%) Group A and $5(16.1 \%)$ of Group B students. Less common mistakes were observed in activation of emergency system and reevaluation of patients. No mistakes were observed in chest compressions.

\section{Discussion}

Based on our findings, a 2-hour class plus a 2-hour practice session on a high-fidelity simulation model, followed by a two-week period of optional additional practice sessions is enough to achieve competence on BCPR in medical students.

Avis, et al. [12] propose that reading and other passive forms of learning provide barely $20 \%$ of retention after 6 weeks, as opposed to active forms of learning, such as simulation, in which retention is as high as $60-70 \%$ after 6 weeks. Our results show retention of $45 \%$ for BCPR in simulation models at 8 weeks.

Wik, et al. [13] found that the most common mistakes in BCPR are those related with ventilation and chest compressions. Our results favor that, as we found the most mistakes in ventilation; nevertheless, second to that we found fail to verify pulse. This may be due to emphasis during training on the importance of chest compressions, as established by AHA in 2010. [8]

International Consensus on Cardiopulmonary Resuscitation and Emergency Cardiovascular Care Science With Treatment Recommendations published by AHA in $2010[8,10]$, suggest that competence on BCPRis lost as soon as 3 months after training. Our findings coincide with that, as we found loss of competence in most participants.

The same consensus suggests that one performance challenge in real patients is the time span between learning in a simulated environment and the opportunity to perform in a clinical scenario. In this study we did not find a significant difference between those students already in clinical practice and those without it, suggesting that being in the clinical scenario does not guarantee the opportunity to practice the ability.
Lofaso, et al. [14] observed that simulation-based medical education is an efficient model to improve development in fundamental clinical skills; nevertheless, including simulation in the curricula does not necessarily improve skills in students. This was also found in our study, in which, despite acquisition of competence for BCPR via a structured training, we showed competence not to be permanent.

In conclusion the competence for BCPR on simulation models prevails in $31 \%$ of medical students 90 days after training. There were no statistically significant differences between students already on clinical practice and those without it. We propose that, besides having clinical practice and including BCPR training in the curricula, students should practice BCPR periodically in simulation models, at least every 3 months, to avoid forgetting competence for BCPR through time.

\section{References}

1. Chakravarthy B, Haar E, Bhatt S, Christopher EM, Shahram L, et al. (2011) Simulation in medical school education: a review for emergency medicine. West J Emerg Med 12(4): 461-466.

2. Goodrow M, Rosen K, Wood J (2005) Using cardiovascular and pulmonary simulation to teach undergraduate medical students: cases from two schools. Semin Cardiothorac Vasc Anesth 9(4): 275289.

3. Jeremy D, Sperling M, Sunday C, Yoon Kang (2013) Teaching medical students a clinic approach to altered mental status: simulation enhances traditional curriculum. Med Educ Online 18: 1-8.

4. Dumas F, Rea T, Fahrenbruch C, Rosenqvist M, Svensson L, et al. (2013) Chest compression alone cardiopulmonary resuscitation is associeated with better long-term survival compared with standard cardiopulmonary resuscitation. Circulation 127(4): 435-441.

5. Hunziker S, Tschan F, Semmer N, Marsch S (2013) Importance of leadership in cardiac arrest situations: from simulation to real life and back. Swiss Med Wkly 143: w13774.

6. Amaya W, Cepeda L, Chaves A (2008) Educación en reanimación básica: Qué tanto y cómo se aprende. Rev Col Anest 36: 33-38. 


\section{Open Access Journal of Pharmaceutical Research}

7. Surcouf J, Chauvin S, Jenelle FTY, Brian B (2013) Enhacing residents neonatal resuscitation competency through unannounced simulation-based training. Med Educ Online 18.

8. Mancini M, Soar J, Bhanji F (2010) International Consensus on Cardiopulmonary Resuscitation and Emergency Cardiovascular Care Science with Treatment Recommendations. Circulation 122: 539581.

9. Wolfram R, Warren C, Doyle C, Kerns R, Frye S (2003) Retention of pediatric advanced life support (PALS) course concepts. J Emerg Med 25(4): 475-479.

10. Soar J, Mancini M, Bhanji F (2003) International Consensus on Cardiopulmonary Resuscitation and Emergency Cardiovascular Care Science with Treatment Recommendations. Circulation 81: 288330.
11. American Heart Association (AHA) (2011) Hoja de prueba de habilidades de SVB/BLS en adultos con $1 \mathrm{y}$ 2 reanimadores con DEA. Libro del instructor de SVB/BLS para profesionales de la salud. 1o edición. Estados Unidos: American Heart Association 23-24.

12. Avis K, Lozano D, White M, Marjorie LW, Amber QY, et al. (2012) High-fidelity simulation training for sleep technologists in a pediatric sleep disorders center. J Clin Sleep Med 8(1): 97-101.

13. Wik L, Myklebust H (2005) Twelve-month retention of CPR skills with automatic correcting verbal feedback. Resuscitation 66(1): 27-30.

14. Lofaso D, De Blieux P, Di Carlo R, Peter MD, Charles H, et al. (2011) Design and effectiveness of a required pre-clinical simulation-based curriculum for fundamental clinical skills and procedures. Med Educ Online 16. 